\title{
RAPIDLY PROGRESSIVE INTERSTITIAL LUNG DISEASE AND HYPOMYOPATHIC DERMATOMYOSITIS
}

\begin{abstract}
Larissa Vargas Cruz ${ }^{1, \star}$, Afonso Guilherme Schmidt ${ }^{1}$, Andrea Worm Furtado ${ }^{1}$, André Lucas Ribeiro ${ }^{1}$, Larissa Martinelli Dullius ${ }^{1}$, Augusto Emílio Hinterholz ${ }^{1}$, Rafael Mendonça da Silva Chakr ${ }^{1}$, Vanessa Hax ${ }^{1}$, Natália Sarzi Sartori ${ }^{1}$
\end{abstract}

1.Hospital de Clínicas de Porto Alegre, Porto Alegre (RS), Brazil.

*Corresponding author: larissavcruz@hcpa.edu.br

\section{BACKGROUND}

Clinically amyopathic/hypomyopathic dermatomyositis is characterized by minimal muscle involvement and the typical cutaneous findings of dermatomyositis. The melanoma differentiation-associated gene 5 (MDA-5) antibodies are found in a proportion of these patients, and are associated with skin ulceration, Gottron papules, hyperkeratosis and severe and rapidly progressive interstitial lung disease, with some case reports of pneumothorax or pneumomediastinum.

\section{CASE REPORT}

A 28-year-old female patient with refractory hypoxemic respiratory failure was transferred to a tertiary hospital for extracorporeal membrane oxygenation (ECMO). Family members reported a previous history of photosensitivity, arthralgia, myalgia and progressive dyspnea, without muscle weakness. Examination revealed heliotrope eruption, Gottron papules with skin ulcer in one metacarpophalangeal joint. Laboratory tests showed ANA 1/80 speckled staining pattern, hyperferritinemia, creatine kinase and lactate dehydrogenase were slightly raised. Myositis-specific autoantibodies, such as anti-MDA-5 were unavailable. Computed tomography chest showed ground glass opacities more than $50 \%$ of the parenchyma and pneumothorax. COVID-19 and other infections were ruled out. Bronchoscopy with bronchoalveolar lavage excluded alveolar hemorrhage. The patient received intravenous steroids, total dose of $5 \mathrm{~g}$, intravenous immunoglobulins and tacrolimus. After 4 weeks in ECMO, she was still extracorporeal circulation dependent, did not show any sign of pulmonary improvement, and there were no signs of infection. She underwent surgical lung biopsy, which showed diffuse alveolar damage in a proliferative phase, with an important component of fibrosis. Unfortunately, she died as a consequence of pulmonary impairment.

\section{CONCLUSION}

Although the presence of anti-MDA-5 antibodies was not confirmed, hypomyopathic dermatomyositis associated with these antibodies was our diagnostic hypothesis, due to the specific cutaneous signs, the severe interstitial lung disease and the exclusion of other possible diseases. The refractoriness to the immunosuppression performed also reinforces this diagnosis, since medical literature discloses that more than half of the patients follow a fatal course. It is important to recognize this rare dermatomyositis subtype because an earlier and aggressive treatment is essential to the outcome. There is limited data, based on observational studies and case reports, to guide the optimal treatment. The recommendations include combination therapy: glucocorticoids and immunosuppressive drugs, such as calcineurin antagonists, and cyclophosphamide as a third drug.

\section{KEYWORDS}

Hypomyopathic dermatomyositis, Anti-MDA-5 antibodies, Interstitial lung disease. 